\title{
Resistencias, novedades y negociaciones: la terminología química durante la primera mitad del siglo XIX en España
}

\section{José Ramón Bertomeu Sánchez y Rosa Muñoz Bello (*)}

(*) Instituto de Historia de la Medicina y de la Ciencia «López Piñero» Jose.R.Bertomeu@uv.es

SUMARIO 1.--Introducción. 2.-La llegada de la nueva terminología. 3.- Controversias. 4.-La renovación constante de la terminología. 5.-Persistencias y resistencias. 6.-Conclusiones.

RESUMEN: En este artículo se analiza la nueva terminología química en España durante la primera mitad del siglo XIX. Pretendemos mostrar que el estudio de la historia de la terminología permite conocer mejor los mecanismos de negociación, apropiación y resistencia de las comunidades locales, y ofrece nuevas claves para repensar las relaciones entre centro y periferias científicas. Las fuentes principales del estudio son los manuales de química publicados entre 1788 y 1845 en España. Estructuraremos la discusión en torno a cuatro temas que forman parte de las cuestiones generalmente analizadas en los estudios sobre la circulación del conocimiento: apropiaciones, controversias, novedades y resistencias.

PALABRAS CLAVE: Libros de texto de química, terminología química, controversias científicas, centros y periferias, circulación de la ciencia.

KEY WORDS: Chemistry textbooks, chemical terminology, scientific controversies, scientific centers and peripheries, knowledge in transit.

\section{Introducción $(*)$}

Durante los años finales del siglo XVIII y principios del siglo XIX la química sufrió una profunda transformación en su terminología. Fue un proceso impulsado por los trabajos de muchos químicos del siglo XVIII,

(*) Este trabajo ha sido posible gracias al proyectode investigación HUM2006-07206-C03-02, este proyecto está integrado en la Red Temática «Lengua y ciencia» (FFI 2009-05433-E). 
que alcanzó su momento culminante con la publicación del Méthode de nomenclature chimique en 1787. Los estudios sobre la nueva terminología en España han estado basados en el análisis de su recepción por parte de la comunidad química y las contribuciones que realizaron algunos químicos españoles a finales del siglo XVIII, en particular, los trabajos de Juan Manuel de Aréjula ${ }^{1}$. En los años noventa se abrieron nuevas posibilidades, gracias a los proyectos de la European Science Foundation, que propiciaron la colaboración internacional y produjeron numerosos estudios sobre casos poco conocidos ${ }^{2}$. En la última década, los estudios procedentes del mundo de la filología han aportado nuevas perspectivas y métodos de trabajo, con proyectos de investigación que han producido toda una serie de tesis doctorales y permitido la creación de grupos pluridisciplinares para el estudio de del lenguaje científico en castellano, por ejemplo, en torno al proyecto CORDE (Corpus Diacronico del Español) ${ }^{3}$.

Teniendo en cuenta todas estas contribuciones, nuestra intención con este artículo es ofrecer una visión diferente del problema a través de un cambio de perspectiva, tanto en la orientación historiográfica como en las fuentes, los actores históricos y el período considerado. Pretendemos mostrar que el estudio de la historia de la terminología ofrece numerosos ejemplos de los mecanismos de negociación, apropiación y resistencia relacionados con la circulación del conocimiento, al mismo tiempo que permite desvelar algunas claves para repensar las cambiantes relaciones entre centros y periferias científicas ${ }^{4}$. Nuestra intención es ampliar las

1. Gago, Ramón; Carrillo, Juan L.uis; García Ballester, Luis. Juan Manuel de Aréjula (1755-1830) y la introducción en España de la Nueva nomenclatura química. Cuadernos de Historia de la Medicina Española. 1974; 13: 273-295. Una revision de los trabajos publicados en Gago, Ramón. The New Chemistry in Spain. Osiris. 1988; 4: 169-192.

2. El resultado fue el libro colectivo de Bensaude-Vincent, Bernadette; Abbri, Ferdinando, eds. Lavoisier in European context. Negotiating a new language for chemistry. Canton: Science History Publications; 1995, incluye un interesante capítulo de Agustí Nieto-Galán, «The French chemical nomenclature in Spain: Critical points, retorical arguments and practical uses», p. 173-191.

3. Garriga, Cecilio et al. Proyecto: la formación de la terminología química en español. En: Brumme, Jenny, ed. La historia de los lenguajes iberrománicos de especialidad. Madrid: Iberoamericana; 2001, p. 105-119. Una lista de publicaciones se encuentra en la página http://www. lenguayciencia.net. Véase también el volumen monográfico de la Revista de Investigación Lingüística. 2008; 11: 7-309.

4. Secord, James E. Knowledge in Transit, Isis. 2004; 95: 654-672. Gavroglu, K. et al. Science and technology in the European periphery: Some historiographical reflections. History of Science. 2008; 46 (2): 153-177. 
fuentes de análisis para prestar mayor atención a obras de gran difusión como los manuales de enseñanza, los libros de divulgación o las reseñas de libros aparecidas en revistas generales. Estas fuentes conducen inevitablemente a aumentar el número de protagonistas históricos, incluyendo, por ejemplo, los autores de los manuales, sus editores y sus destinatarios. La comunidad de personas interesadas por la química a finales del siglo XVIII no era homogénea. Sus diferentes intereses y expectativas moldearon las respuestas frente a la nueva nomenclatura química. Tanto los partidarios como los detractores de las nuevas expresiones tenían razones e intereses que trataremos de analizar de modo simétrico, aprovechando los documentos que nos han dejado las controversias de esos años. Finalmente, para poder tratar estas cuestiones, resulta necesario ampliar el período histórico y avanzar más allá de los años situados alrededor de 1787. Aunque tuvo defensores muy tempranos, el proceso de adopción de la nueva terminología química fue lento y complejo, sin que supusiera el reemplazamiento automático de las viejas expresiones, las cuales presentaban ventajas indudables para determinados usuarios. Por ello, analizaremos obras de la primera mitad del siglo XIX poco estudiadas hasta la fecha. Estructuraremos la discusión en torno a cuatro temas: apropiaciones, controversias, novedades y resistencias.

\section{La llegada de la nueva terminología}

Los intentos de reforma de la terminología química del siglo XVIII culminaron con la obra de Louis-Bernard Guyton de Morveau, Antoine Laurent Lavoisier, Antoine Fourcroy y Claude Louis Berthollet publicada en 1787. Fue la cristalización de diversas propuestas de modificación del vocabulario químico realizadas anteriormente por autores como Pierre Joseph Macquer o Torbern Bergman, las cuales se enmarcaban en el interés ilustrado por construir un lenguaje racional y sistemático para las ciencias, tal y como se puede comprobar en otras influyentes propuestas de reforma del vocabulario científico como la nomenclatura botánica de Lineo ${ }^{5}$. En el campo de la química, la reforma se basaba en la lista de elementos químicos pro-

5. Una revisión general se puede encontrar en Gutiérrez Rodilla, Bertha. La ciencia empieza en la palabra. Barcelona: Península, 1998 y Crosland, Maurice. The language of science: from the vernacular to the technical. Cambridge: Lutterworth Press; 2006. 
puesta por Lavoisier, a los que se atribuyó un nombre único y simple. Las sustancias compuestas eran nombradas con expresiones que indicaban su composición, de modo que lo que anteriormente había sido denominado «aceyte de vitriolo» (por su consistencia y su modo de obtención) pasaba ahora a denominarse «ácido sulfúrico» (lo que indicaba la presencia del elemento «azufre» en su composición). Se abandonaron los nombres basados en características físicas (color y sabor), los modos de preparación o las propiedades terapéuticas.

El proceso de adaptación de la terminología química a las diferentes lenguas europeas fue complejo. Se publicaron escritos polémicos, algunos opuestos a los principios de la nueva terminología y otros dirigidos a realizar nuevas propuestas de términos o reglas de nomenclatura. No obstante, en la mayor parte de los casos, los químicos de cada país optaron por una adaptación de los términos a las diferentes lenguas. Esta adaptación no fue automática y dejó abiertas numerosas posibilidades. En lenguas como el inglés, el portugués o el italiano se adoptaron préstamos más o menos modificados del término francés o de la expresión latina correspondiente. En otras lenguas, como el alemán, se optó por crear calcos mediante raíces de la propia lengua con significado semejante al original francés ${ }^{6}$.

La nomenclatura química fue rápidamente traducida al castellano a principios de 1788 para ser empleada en los cursos que impartía Pedro Gutiérrez Bueno en el Laboratorio Químico de Madrid. El autor afirmaba que, al tratarse de nuevas voces, acuñadas en muchos casos por los autores del Méthode de nomenclature chimique, resultaba imposible buscar expresiones castellanas «igualmente significativas y propias» que estuvieran «autorizadas» por «los mejores Diccionarios de la Lengua, y por los Autores más célebres». Por ello, decidió adoptar las expresiones francesas sin apenas modificaciones, con el fin de hacer el lenguaje de la química «común a todos Países» y «facilitar la comunicación de los trabajos de los profesores y aficionados a esta utilísima ciencia» ${ }^{7}$.

6. Para un panorama general del problema y orientación bibliográfica, veáse García, Antonio; Bertomeu, José R. Nombrar la materia. Una introducción histórica a la nomenclatura química. Barcelona: El Serbal; 1999 y Bertomeu, José R.; García, Antonio. La revolución química: entre la historia y la memoria. Valencia: PUV; 2006.

7. «En vista de esto, solo quedaba el arbitrio de adoptar la voz según se halla en el original, o darle la menor mutación que fuera posible, para no desfigurarla. De ambos medios me he valido, y según he juzgado más conveniente, he dejado unas veces la voz original como estaba, diciendo: acetate, acetite, baryte, molybdate, nitrate, nitrite, etc., con los mismos 
Existen muchas otras pruebas que indican la rápida acogida de la nueva nomenclatura en España. El profesor del Colegio de Cirugía de Cádiz, Juan Manuel de Aréjula, preparaba su propia versión de los nuevos términos pero su tarea se interrumpió por la publicación de la obra de Gutiérrez Bueno y dio lugar a sus conocidas Reflexiones críticas sobre la nueva nomenclatura. En el entorno de la Sociedad Vascongada de Amigos del País, que había establecido una cátedra de química, Trino Antonio Porcel publicó en 1789 un artículo con comentarios críticos sobre los nuevos términos y Valentín de Foronda incluyó en su manual, aparecido en 1791, numerosas tablas con nombres antiguos y modernos. Por su parte, Domingo García Fernández, profesor de química en Madrid y asesor de la Junta de Comercio en asuntos relacionados con la industria química, realizó una nueva versión de la nomenclatura para elaborar su traducción del tratado sobre tintes de Claude Louis Berthollet, que apareció entre 1795 y 1796. Además, Gutiérrez Bueno volvió a reeditar su adaptación de la nomenclatura para uso de los estudiantes que acudían a sus cursos de química en el Colegio de Cirugía de Madrid a principios del siglo XIX. También existen pruebas de un temprano uso de la nueva terminología en los cursos de química impartidos en la Universidad de Valencia. En este caso, los nuevos términos aparecen en noticias de prensa y en facturas de compra de productos químicos, lo que sugiere una incipiente difusión fuera del mundo académico a finales del siglo XVIII ${ }^{8}$.

\section{Controversias}

La rápida recepción de la nueva nomenclatura no debe hacer olvidar la existencia de polémicas y críticas. El caso más conocido son las Reflexiones

caracteres que estaban escritas» Método de la nueva nomenclatura química. Madrid: Sancha; 1788 , p. V. (Reimpresión reciente, con estudio introductorio de Ramón Gago, en Madrid: Fundación Ciencias de la Salud; 1994).

8. Veáse Garriga, Cecilio. La recepción de la nueva nomenclatura química en español. Granzgänge. 1997; 4: 33-48. Garriga, Cecilio. El Diccionario Universal de Física de Brisson (1796-1802) y la fijación lexicográfica de la terminología química en español. En: García Turza, C. et al. Actas del IV Congreso Internacional de Historia de la Lengua Española. Logroño: AHLE, Universidad de la Rioja; 1998, p. 179-190. Más datos sobre los ejemplos mencionados en nuestro trabajo, Bertomeu, José Ramón; Muñoz, Rosa. Azoote y Sulfureto. Debates y propuestas en torno a la terminología química durante la primera mitad del siglo XIX (en prensa). 
de Juan Manuel de Aréjula, que incluían, entre muchas otras propuestas, una nueva expresión para el oxígeno ${ }^{9}$. En realidad, existieron muchas otras obras que incluyeron comentarios sobre la nueva terminología. Los autores y los traductores de los manuales de química tuvieron que discutir la adaptación de las nuevas expresiones a la grafía castellana y acomodar las novedades que se iban produciendo, muchas de ellas no previstas por los autores del Méthode. La situación se complicó con la aparición de traducciones diferentes y propuestas terminológicas contrapuestas que dieron lugar a fuertes controversias. La mayor parte cuestionaban el criterio seguido por Gutiérrez Bueno de adoptar los términos franceses con las menores modificaciones posibles, lo que condujo a la introducción de terminaciones en «-e» (oxîde, baryte, sulfate, acetate, acetite, etc.), propias de la lengua francesa. Otros autores defendieron la necesidad de adaptar las expresiones - y, en particular, las terminaciones - a la índole de la lengua de recepción ${ }^{10}$. Estas discusiones tuvieron lugar en otras lenguas. Por ejemplo, William Nicholson, traductor al inglés de un famoso manual de química de A. Fourcroy, adoptó un planteamiento semejante a Gutiérrez Bueno y decidió mantener las expresiones francesas, sin apenas modificación («oxigene», «carbone», «sulphure»). Por el contrario, James St. John, otro de los traductores de la nomenclatura química al inglés decidió introducir cambios para que las nuevas expresiones fueran «agreeable to the custom of English authors» y, por ejemplo, decidió escribir «oxygen» y no «oxigen», para mantener el modo habitual de transcribir la letra ípsilon en inglés. El debate se mantuvo durante algunos años y dio lugar a pequeñas variaciones gráficas en los manuales ingleses ${ }^{11}$.

El debate sobre la mayor o menor adaptación de los términos a la grafía castellana se inició pronto. Aparece ya señalado por Trino A. Porcel en una memoria en la que criticaba las expresiones adoptadas por Gutiérrez Bueno y sugería expresiones como «carbonato, sulfato, nitrato», argumentando que «la terminación en «-o» conviene más con el índole de nuestra lengua que

9. Veáse Gago, Ramón; Carrillo, Juan L. La introducción de la nueva nomenclatura y el rechazo de la teoría de la acidez de Lavoisier. Málaga: Universidad; 1979, que contiene la obra original de Aréjula de 1788.

10. Sobre esta cuestión, veáse Garriga, n. 8, p. 46-47;

11. Veáse Crosland, Maurice P. Historical studies in the language of chemistry. London: Heinemann; 1962, p. 193-194. Más información en Knight, David M. Crossing the channel with the new language. In Bensaude-Vincent, Abbri, n. 2, p. 148-151, que ofrece más ejemplos de lo que denomina «pedantic criticisms». 
la terminacion en «-e» ${ }^{12}$. Los puntos de vista de Gutiérrez Bueno contaron con el respaldo de algunos autores como, por ejemplo, el traductor de la importante obra del sueco Torbern Bergman (1794), que defendió las terminaciones en «-e», señalando otros ejemplos ya consolidados como «calabazate de azúcar», «verdete» $\mathrm{o}$ «mazacote» ${ }^{13}$.

El principal crítico de la postura de Gutiérrez Bueno fue Domingo García Fernández que elaboró una nueva versión de la nomenclatura química para su traducción de los Elementos del arte de teñir de Claude Louis Berthollet. García Fernández señalaba que su traducción se apartaba de la ya publicada «en algunas cosas acerca de las terminaciones de las voces», porque consideraba que debía adoptar sufijos que fueran «conformes á la índole de nuestra lengua y a las intenciones de los autores franceses». De este modo, García Fernández prefería las terminaciones en «-o» para los metales, «oxîdo» en lugar de «oxîde», aunque mantenía la terminación en «-ate» para las sales ${ }^{14}$. Similares puntos de vista defendió Pedro María Olive, traductor de la obra de Antoine Fourcroy, que amplió el uso de las terminaciones en «-o» a las sales («por ser la más propia terminación de nuestro género masculino») y a los metales, con la excepción de «plata», porque, según afirmaba, no se atrevió a introducir el cultismo «argento» ${ }^{15}$. A pesar de las críticas, las terminaciones francesas siguieron siendo empleadas en traducciones tan importantes como las del gran tratado de Louis-Jacques Thenard o el Manual de Química Aplicada a las Artes de Jean-Antoine Chaptal, ambas aparecidas a finales de la segunda década del siglo XIX. La difusión de las terminaciones en «-o» fue impulsada con la traducción del manual de Mateu Orfila, y con la publicación de la obra terminológica de Caventou, traducida por Higinio A. Lorente, ambas aparecidas en 1818. No obstante, en 1827 José Luis Casaseca todavía señalaba que no había «reglas fijas en castellano acerca del lenguaje químico, puesto que unos dicen sulfato y otros

12. Porcel, Trino Antonio. Minas de Hierro. Extractos de las Juntas Generales celebradas por la Real Sociedad Bascongada de los Amigos del Pais. Vitoria: Baltasar de Manteli, 1789, p. 45.

13. Bergmann, Torbern O. Elementos físico-químicos de la analísis general de las aguas... Madrid: Imprenta Real; 1790, p. xli-I. Estas terminaciones aparecen también en otras obras de esos años como las versiones castellanas de los manuales de Fourcroy y Chaptal aparecidas en la última década del siglo XVIII y por supuesto, en las obras de Gutiérrez Bueno de esos años.

14. Bertholet, Claude Louis. Elementos del arte de teñir. Madrid: Imprenta Real; 1795-1796, p. xivXV.

15. Fourcroy, Antoine. Sistema de conocimientos químicos. Madrid: Imprenta Real; 1803, p. vii (prólogo del traductor). 
sulfate». Es fácil encontrar expresiones como «oxides» en obras publicadas en la década siguiente, aunque paulatinamente fueron reemplazadas por las correspondientes expresiones con terminación en «-0» ${ }^{16}$.

El debate sobre las terminaciones produjo un buen número de variantes morfológicas y obligó a los participantes en el debate a hacer explícitos sus puntos de vista en materia terminológica. En la segunda edición de su versión de la nomenclatura, Gutiérrez Bueno se defendía de las críticas recibidas, que consideraba «pueriles», similares a las disputas escolásticas, y afirmaba la necesidad de mantener su traducción literal de los nuevos términos señalando los peligros de la introducción de variaciones terminológicas que podían «dar lugar a la confusion en el lenguage químico» ${ }^{17}$. Desde un punto de vista convencionalista, similar al adoptado por otros autores de esos años, Gutiérrez Bueno pensaba que los nombres de las sustancias no debían ser objeto de polémica. No le parecía relevante la etimología de los nombres, ni los fundamentos que habían servido para acuñarlos. Una vez adoptados lo importante era que sirvieran para designar unívocamente a las sustancias:

«Por esta causa no tengo por conveniente hacer la menor alteración en cuanto a los nombres recibidos en mi escuela; y más quiero que se sepa en ella, qué es oxîgeno, que los fundamentos que han tenido algunos para darles distintos nombres. El que comprehenda bien sus propiedades, entenderá fácilmente cuantos nombres se le quieran dar, con tal que estén bien fundados en ellas» ${ }^{18}$.

Gutiérrez Bueno era consciente de los diversos criterios que se habían seguido para acuñar los nombres de los elementos, por lo que prefería sacrificar la coherencia etimológica en beneficio de la univocidad y la universalidad del lenguaje. Todo ello comportaba aceptar cierto grado de convencionalismo y olvidar la pretensión de que los nombres respondieran sistemáticamente a las interpretaciones consideradas correctas en su

16. La cita de Casaseca procede de su traducción de Magendie, François. Formulario para la preparación y uso de varios medicamentos nuevos. Madrid: Imprenta de D. José del Collado; 1827, p. xv. Más información sobre esta obra y las otras mencionadas en nuestro artículo Bertomeu; Muñoz, n. 8 (en publicación).

17. Gutiérrez Bueno, Pedro. Nomenclatura química para el uso de su escuela pública. $2^{\text {a }}$ ed Madrid: Sancha; 1801, p. i-iii.

18. Gutiérrez, n. 17, p. iii. 
tiempo. En Gran Bretaña, Priestley, Cavendish y otros químicos británicos habían criticado los riesgos que se derivaban de la asociación del lenguaje con una interpretación de los fenómenos químicos que algunos de ellos no compartían, lo que podía dar lugar a una proliferación de tantos nombres como teorías o, más probablemente, a cambios en la terminología a medida que se fueran revisando las conclusiones de los experimentos. De hecho, como veremos más adelante, la aparición de nuevas sustancias, o la reconsideración del carácter elemental de algunas, supuso la constante revisión de una terminología química basada en el análisis y la composición ${ }^{19}$.

La adopción de criterios coherentes resultaba también complicado para los traductores. Aunque Gutiérrez Bueno prefirió mantener un lenguaje casi idéntico a la versión francesa, se apartó de esta tendencia general en casos como «azoote» (para evitar la homonimia con «azote») o «sulfurete» (siguiendo el modelo de la expresión latina «sulphuretum»). La primera expresión tuvo escaso éxito y fue rápidamente reemplazada por «ázoe», la cual fue paulatinamente abandonada a mediados del siglo XIX con el retorno de la expresión «nitrógeno». La segunda expresión, que fue apoyada por críticos como García Fernández, pervivió en los manuales hasta el primer tercio del siglo XIX, cuando fue paulatinamente reemplazada por «sulfuro». También en estos casos los autores discutieron sobre la necesidad de introducir estas novedades, así como sobre los procedimientos de adaptación de las voces al castellano y su mayor o menor semejanza con las correspondientes expresiones francesas ${ }^{20}$.

Todas estas cuestiones afloraron a lo largo de la polémica sobre las terminaciones que continuó vigente hasta la década de 1820. Una reseña del manual de Mateu Orfila aparecida en la revista Crónica Científica y Literaria protestaba por la mala calidad de la versión castellana, hasta el punto de afirmar que la traducción no podía ser obra del propio Orfila, tal y como se afirmaba en el título ${ }^{21}$. Con argumentos que recordaban los utilizados por Gutiérrez Bueno, señalaba el peligro de introducir novedades no justificadas

19. Sobre esta cuestión, veáse el apartado 4. La renovación constante de la terminología. Las opiniones de los autores británicos son analizadas por McEvoy, John; Priestley, G. Responds to Lavoisier's nomenclature. En: Bensaude-Vincent; Abbri, n. 2, p. 123-142, especialmente pp. 129-136. Veáse en este mismo libro el trabajo de Knight, n. 11 p. 143-154. Veáse también los puntos de vista de Vicenzo Dandolo en Beretta, Marco. Italian traslations of the Méthode de Nomenclature Chimique. En: Bensaude-Vincent; Abbri, n. 2, p. 225-247.

20. Véase Bertomeu; Muñoz, n. 8.

21. Critica. Crónica Científica y Literaria. 1818; 116: 1. Está firmado por A. O. 
en la terminología. El autor afirmaba que resultaba lícito formar nuevas palabras cuando se descubría un cuerpo nuevo pero «una vez adoptado y generalizado» no debía variarse «sin ventajas muy notables y motivos muy poderosos». Y, por ello, protestaba por haberse mudado en la traducción las voces «bore, clore, iode y flore» al cambiarles «la e final en o», logrando así «no sólo mudar el aspecto de estas voces, sino también hacerlas mucho más duras y desagradables». Defendía, por el contrario, «la armonía y facilidad [...] de estos nombres adoptados con su terminación en e», reforzados por «la práctica constante, pues que así se nombran en todas las cátedras de enseñanza, y en las pocas obras impresas en castellano que hablan de estos cuerpos». Estos comentarios revelan la gran difusión que habían obtenido las nuevas voces desde su introducción por Gutiérrez Bueno en 1788. No obstante, el revisor también recordaba que «algunos escritores españoles» empleaban las expresiones «oxidos, sulfatos y fosfatos» y acusaba al autor de la traducción de falta de coherencia, porque en este caso había optado por la terminación en «-e» (nitrates, sulfates, fosfates, etc.) ${ }^{22}$.

\section{La renovación constante de la terminología}

Los comentarios críticos a la traducción del manual de Orfila muestran las dificultades terminológicas producidas por la constante renovación de los conocimientos químicos, el aislamiento de nuevas sustancias elementales y el incremento exponencial del número de compuestos conocidos en las primeras décadas del siglo XIX. En previsión de esta situación, los autores del Méthode habían sugerido una serie de reglas que permitían nombrar tanto los compuestos conocidos como los que pudieran descubrirse en el futuro. Pero estas reglas no eran suficientes para todos los casos y, a medida que avanzó el siglo XIX, aparecieron nuevas sustancias que no se ajustaban a ellas. Ya a principios del siglo XIX, el químico británico Richard Chenevix (1774-1830) afirmaba que la química precisaba de «una completa revisión de la nomenclatura», para lo que proponía nombrar una comisión formada por un grupo selecto de químicos ${ }^{23}$.

\footnotetext{
22. Critica. Crónica Científica y Literaria, n. 21, p. 2.

23. Chenevix, Richard. Remarks upon chemical nomenclature. According to the principles of the French neologist. London: J. Bell; 1802, p. 191-192. Citado por Crosland, n. 11, p. 213. Veáse Knight, n. 11, p. 149-150.
} 
En el campo de la química vegetal y animal apenas tuvo vigencia la aplicación de los nombres diseñados por los químicos franceses. Los nuevos alcaloides descubiertos en las primeras décadas del siglo XIX fueron nombrados mediante procedimientos tradicionales, a partir de los nombres de las plantas de las que se extraían o de sus propiedades terapéuticas, ya que resultaba imposible emplear la composición química como criterio principal. No obstante, dado que el número de sustancias era reducido, la terminología así acuñada resultó manejable hasta la llegada de la nueva química orgánica en la segunda mitad del siglo XIX. En la química mineral, las dificultades se centraron en torno a los compuestos formados por los mismos elementos en diversas proporciones, un problema que afectaba especialmente a los óxidos y sus sales. Los creadores del Méthode no desarrollaron demasiado sus propuestas en este campo, puesto que se conocían pocos compuestos de estas características a finales del siglo XVIII. Así, por ejemplo, diferenciaron entre «oxide de fer rouge» $\mathrm{y}$ «oxide de fer noir» (para los dos óxidos conocidos del hierro) y entre «muriate de mercure corrosif» $\mathrm{y}$ «muriate de mercure doux» (para lo que actualmente denominaríamos cloruros de mercurio). Como puede comprobarse, la distinción entre estos compuestos binarios formados por los mismos elementos en diversas proporciones se basada en los mismos criterios que la nomenclatura tradicional anterior a 1787: el color, el sabor, las propiedades médicas, etc. Para solucionar este problema, se realizaron varias propuestas que fueron recogidas posteriormente en los manuales de química. Las más importantes fueron introducidas por Joseph-Louis Proust, Thomas Thomson, Louis-Jacques Thenard y Jöns Jacob Berzelius.

En la década de 1790, Joseph-Louis Proust introdujo las expresiones latinas ad maximum y ad minimum para indicar la proporción de oxígeno en los compuestos del hierro. Los nuevos términos fueron introducidos por Proust en sus trabajos sobre el azul de Prusia para diferenciar distintos grados de oxidación del hierro y aparecieron a finales de la década de 1790 en diversas publicaciones que realizó en francés y en castellano ${ }^{24}$. Las ideas terminológicas de Proust tuvieron amplia difusión en las obras españolas de las dos décadas siguientes. Resultaban especialmente interesantes para sales formadas por los mismos metales en distinto estado de oxidación, tal y 
como ocurría con algunas sustancias empleadas como medicamentos, lo que explica la rápida acogida de estas expresiones en obras de farmacia ${ }^{25}$.

En su famoso tratado System of Chemistry, Thomas Thomson criticó el uso del color para distinguir las sustancias y propuso emplear prefijos con la «primera sílaba de los numerales griegos», de modo que el «protoxide» sirviera para indicar el metal combinado con el mínimo de oxígeno, el «deutoxide» el siguiente con más oxígeno y así sucesivament hasta el «peroxide», que designaba al metal «completamente oxidado» (thoroughly oxidised ${ }^{26}$. Las ideas de Thomson llegaron a través de las versiones del gran tratado de química de Louis-Jacques Thenard que fue una de las obras más influyentes de la primera mitad del siglo XIX. Esta obra fue rápidamente traducida para las lecciones impartidas en el Real Laboratorio de Madrid. Se preparó una versión reducida y notablemente modificada del original, donde el traductor J. Acosta, ayudante del Real Laboratorio, se apartó bastante de la nomenclatura sugerida por Thenard, la cual sustituyó, en ocasiones, por propuestas anteriormente realizadas por Joseph-Louis Proust. En el apartado dedicado a la «nomenclatura», donde se discutían los nombres de los «óxîdes» con diversas proporciones de oxígeno mediante el empleo de prefijos, el traductor añadió una nota donde aclaraba que esta terminología, basada en prefijos griegos, se debía a Thomson pero recordaba que Proust había sido el primero en dar respuesta a este problema ${ }^{27}$. A lo largo de la obra, el traductor incluyó muchas referencias a los nombres sugeridos por Proust, comparándolos con los que ofrecía Thenard y proponiendo algunos cambios ${ }^{28}$.

Como vemos, el traductor de la obra de Thenard introdujo notables modificaciones en cuestiones terminológicas, adoptando expresiones propuestas previamente por Proust, el anterior director del Laboratorio Químico de Madrid. El ejemplo muestra el amplio margen que disponían los traductores para ayudar a difundir nuevas voces o alargar a la persis-

25. Bañares, Gregorio. Filosofía Farmacéutica o la farmacia reducida a sus verdaderos principios. Madrid: Imprenta Real; 1804, p. 80-81 y en la nueva edición de Madrid, 1814, p. 100-101.

26. Crosland, n. 11, p. 217.

27. Lecciones elementales de química teórica y práctica. Madrid: Imprenta Real; 1816-1819, vol. 1, p. 163-164.

28. Lecciones, n. 27, vol. iii, p. 141-143. Al tratar del «peróxide» de níquel señala en nota se indica que se trata del «deutóxide de Thenard y óxide mayor de Proust». También ocurre en otros casos como el «peróxide de mercurio», del que se dice que es el «deutóxide de Mr. Thenard». 
tencia de voces antiguas. Todo ello contribuyó a aumentar el número de sinónimos existentes para designar una sustancia. Por ejemplo, el profesor del Real Laboratorio de Química de Madrid, Juan Mieg, publicó en esos años unas lecciones, donde empleaba la expresión «oxîdulo» con la que designaba «en general un grado muy débil de oxidación», si bien reconocía que «en el día» se empleaban con este mismo fin «los nombres de oxîde ad minimum ó proto-oxîde» ${ }^{29}$. La expresión «oxîdulo» era un diminutivo empleado en Francia y Alemania para designar los compuestos con menor proporción de oxígeno pero, como el propio Mieg señalaba, había quedado obsoleta cuando «los químicos han reconocido que algunas substancias metálicas son susceptibles de unirse con el oxîgeno en mas de dos proporciones» ${ }^{30}$. Esta crítica era también aplicable a las propuestas de Louis Proust que sólo permitían nombrar dos óxidos formados por una sustancia con más («ad maximum») o menos («ad minimum») oxígeno. Por ello, otro autor de manuales de esos años, el licenciado en farmacia Miguel Piñol i Pedret, sugería abandonar las expresiones de Proust y abrazar las nuevas propuestas de Thomson y Thenard. En sus comentarios se puede observar la diversidad de nombres de óxidos que debían manejar los químicos españoles en esos años:

«para manifestar los grados de oxigenacion que reciben los cuerpos orgánicos e inorgánicos, los Químicos españoles se valen de las demostraciones propias del mismo idioma, como es primer grado, segundo, tercero, cuarto, etc. y tambien de los nombres derivados del idioma latino ad minimum y ad maximum y las terminaciones en oso para la denominacion de los ácidos débiles; en ico para la de los ácidos mas oxigenados, y de sobre oxigenados para la de los ácidos con su mayor oxigenacion: los Químicos franceses se valen para determinar estos grados de oxigenacion de las terminaciones protóxidos, deutóxidos, tritóxidos, tetróxidos, etc., y al mayor grado de oxigenacion, peróxidos, derivados sin duda del idioma griego» ${ }^{31}$.

Piñol consideraba estas últimas denominaciones «conforme[s] al estado actual de la ciencia» debido a la existencia de «metales [que] reciben hasta cinco grados diferentes de oxidación», lo que hacía que las denominacio-

29. Mieg, Juan. Lecciones elementales de química para uso de los principiantes. Madrid: Catalina Piñuela; 1816 , p. 216-217

30. Mieg, n. 29, p. 257-258.

31. Piñol, Miguel. Elementos de física-química. Madrid: Villalpando; 1820, p. 30-31. 
nes ad minimum y ad maximum hubieran perdido validez ${ }^{32}$. Las nuevas denominaciones de Thomson y Thenard fueron difundidas a través de la traducción de la obra terminológica de Joseph Bienaimé Caventou, plenamente dedicada a presentar la nueva nomenclatura y las clasificaciones de Thenard, con una larga lista de sinónimos ${ }^{33}$.

No todos los autores aceptaron las ideas de Thenard. El farmacéutico Gregorio Bañares consideraba innecesarios los nuevos prefijos griegos y sugería que, en caso de necesidad, se podrían emplear «voces castellanas que no necesitan explicacion preliminar», por ejemplo, «en lugar de protoxido hubiera dicho primeroxido, en el de deutoxido, segundoxido, y en el peroxido, ó polioxido, mayoroxido» ${ }^{34}$. Sus ideas apenas tuvieron eco y fueron pronto criticadas por Manuel Jiménez en su nomenclatura farmacéutica de $1826^{35}$. Otra propuesta más original y coherente, aunque también con escasa difusión, fue realizada en esos años por otro farmacéutico, Antonio Chalanzón, en una obra dedicada a una extensa revisión de la nomenclatura química. Su intención era presentar una nomenclatura «en los que los nombres genéricos de las combinaciones binarias en sus diferentes proporciones» fueran todos «unívocos»y, al mismo tiempo, reducir el tamaño de las expresiones que designaban las sales. De este modo, pretendía que las voces «sulfuríto», «sulfureto» y «sulfuro» se emplearan para expresar combinaciones de diversas proporciones de azufre con un mismo elemento, del mismo modo que proponía «oxidítos, oxidulos y oxídos» para diversos compuestos de un metal en diverso grado de oxigenación, lo que conducía a abandonar los prefijos de Thomson y Thenard $^{36}$.

\footnotetext{
32. Piñol, n. 31, p. 31

33. Caventou, J. B. Nueva nomenclatura química... Madrid: Calle de la Greda; 1818

34. También proponía reemplazar el prefijo «per» por «poli». Bañares, Gregorio. Análisis del agua mineral de los baños de la Fuensanta ó hervideros... Madrid: Núñez de Vargas; 1820, p. 15.

35. Jiménez Murillo, Manuel. Nomenclatura farmacéutica, y sinonimia general de Farmacia y Materia Médica. Madrid: Eusebio Alvárez; 1826, p. v-vi, indica que seguirá la formación de óxidos con los prefijos «deuto-», «tri-», «per-» y rechaza la voz sugerida por «Bañares» de «polióxido» porque considera que «como esta voz significa mezcla de muchos óxidos, y no los metales en el último grado de oxidacion, nos es imposible admitirla» (p. vi).

36. Chalanzon, Antonio. Historia natural, análisis y virtudes del agua mineral ferruginosa de la fuente sublantina... Leon: Imprenta de D. Pablo Miñon; 1821, p. 40-41. La explicación de Chalanzón es suficientemente ilustrativa: «si á un sulfureto cualquiera se le añade cierta cantidad de metal, el producto será un Sulfurito, y añadiéndole Azufre, convertiremos el Sulfureto en un Sulfuro, etc.». Analizaremos con más detalle esta interesante propuesta terminológica en un próximo trabajo.
} 
El problema causado por los largos nombres de las sales, señalado por Chalanzón, preocupó a muchos autores de esos años. Para reducir las expresiones, Thenard proponía colocar el prefijo correspondiente (proto-, deuto-, trito-, etc.), junto al nombre del ácido, de modo que en lugar de «sulfate de protoxide de mercure», podía escribirse abrevidamente "proto-sulfate de mercure» ${ }^{37}$. Esta simplificación fue también objeto de debate porque producía confusiones. José Luis Casaseca, un discípulo de Thenard, escribía en 1828 que «algunas de estas abreviaciones tienen el inconveniente de dar una idea equivocada de la composición de estas sales, pues parece que las expresiones proto, deuto, etc. se refieren al ácido y no al óxido», de modo que parecían expresar «combinaciones de una, dos y tres proporciones de ácido con la misma cantidad de óxido», por lo que Casaseca recomendaba que «no deberian usarse» ${ }^{38}$. Estas cuestiones afectaban directamente al grupo de las sales que era el más numeroso dentro de las sustancias inorgánicas. El propio Thenard era consciente de la gravedad del problema y, para solucionarlos, sugería un gran acuerdo entre los químicos de todas las naciones que nunca llegó a producirse. La llegada de las nuevas expresiones sugeridas por Berzelius complicó más la situación hasta que finalmente acabaron imponiéndose a partir de $1840^{39}$.

Como vemos, la diversidad terminológica no estuvo únicamente propiciada por las decisiones tomadas por los traductores. Las constantes novedades terminológicas propiciaron la coexistencia de una multiplicidad de sinónimos porque no supuso la eliminación de las expresiones antiguas $\mathrm{y}$, como hemos visto, algunos traductores decidieron mantener formas de nombrar que estaban bastante difundidas gracias a la presencia de su autor, Joseph-Louis Proust, al frente del Laboratorio de Madrid. También hemos visto que existieron críticas a las nuevas propuestas e, incluso, propuestas originales, como las elaboradas por Antonio Chalanzón y Gregorio Bañares. El hecho de que ambos autores fueran farmacéuticos no es casual porque, como veremos en el siguiente apartado, las principales resistencias a la nueva nomenclatura procedieron de este grupo profesional.

37. Thenard, Louis Jacques. Traité de chimie élémentaire, théorique et practique. Paris: Crochard; 1813-16, vol. 2, p. 293-297.

38. Sobre esta cuestión, veáse Crosland, n. 11, p. 220.

39. Thenard, Louis Jacques. Tratado completo de química teórica y práctica. Nantes: Busseil y Compañía; 1830, vol. 1, p. 20-22, donde Thenard discute muchos aspectos de la terminología y revisa algunos de sus puntos de vista anteriores. No podemos tratar aquí la introducción de la nomenclatura de Berzelius, un tema al que dedicaremos un futuro trabajo. 


\section{Persistencias y resistencias}

Aunque fue pronto conocida, no todos los autores aceptaron de buen grado los nuevos términos químicos del siglo XVIII. Lógicamente, el problema afectaba sobre todo a las personas formadas en la química del siglo que habían aprendido los términos antiguos. Un lector anónimo se preguntaba en 1789 en la Gaceta de literatura de México sobre la suerte de las obras de química escritas antes de la reforma terminológica:

«Ya habrá V. visto la nueva nomenclatura química ¡Qué trastorno! ¿Qué nuevo trabajo y muy reduplicado se presenta a los que intenten cultivar esta bella ciencia? ¿Qué haremos con las obras de los Stales, Boheraves [sic] y de otros muchos a cuyas fatigas, a cuyos descubrimientos debemos las verdades químicas de que nos gloriamos? ¿Se reimprimirán con arreglo á la nueva nomenclatura? ¿Se reimprimirán en el estado que las publicaron sus autores? Si lo primero, resultaran desperfeccionadas e ininteligibles; si lo segundo, nada hemos abanzado de útil, porque será necesario recargar la memoria conservando dos espresiones para reconocer un solo objeto» ${ }^{40}$.

Además de apuntar el corte con el pasado que introducía la nueva terminología, el perspicaz lector apuntaba ya uno de los problemas que, como hemos visto, se agudizó en los años siguientes: la multiplicidad sinonímica. Estas críticas generales contra la nueva nomenclatura debieron ser más habituales de lo que las fuentes históricas hasta ahora analizadas permiten concluir. Todavía en 1818, el anónimo autor de la ya mencionada reseña del manual de Orfila, escribía que «diferentes veces» había oído «declamar contra la nomenclatura química, contra las innovaciones que en ella se hacen, y contra el abandono de las voces antiguas», aunque consideraba que esta crítica era «absolutamente infundada para todo el que haya tomado gusto á esta ciencia» ${ }^{41}$. Sea como fuere, lo que sí podemos constatar con total certeza es la permanencia de voces antiguas muchas décadas después de la llegada de la nueva nomenclatura. Además de las fuentes ya citadas, podemos añadir una versión revisada de la filosofía química de Fourcroy que apareció muy tardiamente (1827) en la Habana

\footnotetext{
40. Carta al autor de esta Gaceta. In: Alzate Ramírez, José Antonio. Gacetas de literatura de México. Puebla: Hospital de S. Pedro; 1831, vol. 1, p. 92-99. Está firmada por «Pedro el observador» y fechada en 7 de enero de 1789.

41. Crónica Científica y Literaria, n. 21.
} 
con una «novísima nomenclatura química» donde se marcaban con un símbolo especial los nombres antiguos que «se usan todavía en el lenguaje químico». Entre ellos figuraban «aceite de vitriolo», «ácido borácico», «ácido prúsico», «agua fuerte», «alumbre», «azul de Prusia», «cinabrio», «crémor de tártaro», «emético», «espíritu de vino», «ethiope marcial», «manteca de antimonio», «mercurio dulce», «minio», «plombagina», «salitre», «sal de acederas», «sal de saturno», «sublimado corrosivo» o «vitriolo» (azul, blanco, verde), etc. ${ }^{42}$.

Un pequeño repaso a la lista anterior permite fácilmente comprobar que la persistencia de las expresiones antiguas estuvo relacionada con el uso de estos productos en el mundo de la medicina, la farmacia y la industria. En estas áreas, existían modos de nombrar tradicionales que respondían a las necesidades propias de cada actividad, es decir, las expresiones indicaban aspectos como el color («ethiope marcial»), la consistencia («manteca de antimonio»), el origen («sal de acederas») o las propiedades médicas («emético») que tenían gran relevancia para médicos, farmacéuticos y artesanos. La persistencia de expresiones antiguas en estas áreas fue un hecho generalmente asumido como inevitable por los autores de los libros de texto de química. En su traducción de la obra de Berthollet, Domingo García Fernández señalaba:

«Se extrañará tal vez, que siendo Berthollet uno de los fundadores de la nueva nomenclatura Chîmica se use en la presente obra promiscuamente de las antiguas denominaciones y de las modernas; pero si se reflexîona, se hallará que se ha visto precisado á tomar este partido en virtud de que su obra habla con los artistas y los sabios, y que ha querido satisfacer á todos. No me he atrevido á alterar cosa alguna sobre este asunto, y solo me he tomado la libertad de insertar la nueva Nomenclatura moderna en una columna y la antigua en otra, en beneficio de los que no estén enterados de ellas» ${ }^{43}$.

Otro de los máximos defensores de la nueva terminología en Francia, Jean-Antoine Chaptal, había realizado también consideraciones semejantes en su manual de química, con el fin de que sus contenidos fueran compren-

42. Fourcroy, Antoine. Filosofía química ó verdades fundamentales de la química. Habana: Díaz de Castro; 1827, p. 139-144.

43. Berthollet, Claude Louis. Elementos del arte de teñir, escritos en francés por Monsieur Berthollet (traducidos al castellano por D. Domingo García Fernández). Madrid: Imprenta Real; 17951796, p. xiv-xv. 
sibles para los artesanos a los que se dirigía la obra y, de este modo, vencer los recelos sobre las posibles aplicaciones de la química en las artes ${ }^{44}$. Por ello, en muchas de las obras dirigidas a este público, como la escrita en París por los dos pensionados Garriga y San Cristóbal, se mantuvieron los sinónimos antiguos junto con las voces modernas que eran, por regla general, las más empleadas a lo largo del texto ${ }^{45}$.

Una situación similar se produjo en las obras dirigidas a médicos, cirujanos y boticarios, si bien, en esta ocasión, resulta más fácil obtener testimonios de las posturas críticas ante la nueva nomenclatura. La nueva nomenclatura fue rápidamente conocida en esta área, gracias a los cursos de química que se impartían en diversas instituciones dirigidas a su formación. Por ejemplo, las obras y las lecciones de los boticarios Pedro Gutiérrez Bueno en Madrid y de Francesc Carbonell i Bravo en Barcelona que contribuyeron a la rápida difusión de la nueva terminología química en el mundo de la farmacia. Un discípulo de Gutiérrez Bueno, Antonio de la Cruz, mencionó la nueva nomenclatura en un ejercicio de 1788, aunque reconocía que era todavía poco conocida ${ }^{46}$. Carbonell i Bravo defendió la reforma de la terminología farmacéutica en una memoria de 1798 y la adoptó en su popular manual de farmacia, que tuvo numerosas ediciones ${ }^{47}$. No resulta difícil encontrar la presencia de los nuevos términos en las obras escritas o traducidas por farmacéuticos de finales del siglo XVIII y principios del siglo XIX. Esta rápida difusión explica que la nueva

44. Chaptal, Jean Antoine. Elementos de química. Madrid: Viuda e hijos Marín; 1793-1794, vol. 1, p. 22.

45. San Christóbal, J. M.; Garriga y Buach, J. Curso de química general aplicada a las artes. Paris: Carlos Crapelet; 1804-1805.

46. Memorial literario. 1788; 14: 289-290.

47. Su memoria se leyó en la Real Academia Médico-Práctica de Barcelona en el año 1798, con el título de «Reflexiones sobre la nueva nomenclatura química». Carbonell i Bravo, Francisco. Elementos de farmacia, Barcelona, Texeró, 1824, p. 148. En 1802 defendió una «reforma sistemática en la nomenclatura de la Farmacia», en la línea de la nueva terminología química que, según este autor, había producido «grandes ventajas en el estudio de la chîmica [...] ya facilitando aquel estudio por la simplicidad y exâctitud de las voces, ya desterrando las falsas ideas que imprimian las antiguas impropias denominaciones». Carbonell i Bravo, Francisco. Elementos de farmacia, Barcelona: Piferrer; 1802, p. 132. Sobre este autor, véase Nieto-Galan, Agustí. Ciència a Catalunya a l'inici del segle XIX: teoria i aplicacions tècniques a l'escola de Química de Barcelona sota la direcció de Francesc Carbonell i Bravo (1805-1822). Barcelona: Tesis doctoral, 1994; Francesc Carbonell i Bravo, Discurs d'obertura de l'Escola de Química de Barcelona, 1805 (Introducció a cura d'Agustí Nieto-Galan). Barcelona: SCHCT; 2005. 
farmacopea hispánica, que se publicó en 1794, recogiera ya algunas de las nuevas expresiones, aunque siempre señalando su correspondencia con las voces antiguas 48 .

Estas referencias no deben hacer olvidar, sin embargo, muchos otros libros que mantuvieron las viejas expresiones, sin apenas señalar la existencia de las voces nuevas, incluso bastantes años después de su creación. Los autores que emplearon la nueva nomenclatura estuvieron siempre obligados a mencionar las expresiones antiguas u ofrecer tablas de sinónimos, lo que indica que las nuevas voces se abrieron paso muy lentamente. En 1804, Gregorio Bañares criticaba el estado de la nomenclatura farmacéutica pero reconocía que había tenido que «conservar hasta mejor ocasión los nombres antiguos (...) por no tener idea de semejante nomenclatura la mayor parte de los profesores» de medicina, cirugía y farmacia ${ }^{49}$. Otra prueba de la persistencia de las voces antiguas es la aparición de varias obras como la que realizó el boticario Antonio de la Cal en 1811, con el fin de facilitar «la inteligencia de los nuevos Nombres (...) por medio de la adición de los nombres con que han sido siempre conocidos y se conocen, y con que se piden comunmente en sus recetas por todos los Médicos y Cirujanos», lo que, según el autor, debía evitar «equivocaciones perjudiciales (...) en que fácilmente podrían incurrir en su despacho los boticarios no habituados a semejantes nombres nuevamente introducidos». Antonio de la Cal recordaba en su escrito que la «Nueva Nomenclatura Chîmica» era solamente empleada para recetar medicamentos «por un corto número de profesores, especialmente extranjeros» ${ }^{50}$. Con el visto bueno del censor, la obra pudo ser publicada y en el anuncio que apareció en la Gaceta de Madrid,

48. Pharmacopea Hispana. Editio Altera. Matriti: Ex Typographia Ibarriana; 1797. La obra está escrita en latín, recoge bastantes nuevas expresiones y un índice de voces nuevas, con los correspondientes sinónimos tradicionales. No obstante, en muchos apartados, solamente se emplea la terminología tradicional, por ejemplo, «argentum causticum» (vulgò Lapis infernalis); «ferrum vitriolatum» (vitriolum Martis); «rubigo ferri» (crocus Martis). Cuando se hace uso de la nueva nomenclatura, se indica el correspondiente sinónimo tradicional: «hydrargyrus sulphuratus» (Aethiops mineralis), Hydrargyrus Nitratus (Mercurius ruber); «antimonium muriatum» (Butyrum Antimonii), «antimonium sulphuratum rubrum»(kermes minerale); «Tartarus antimonialis» (Emeticus).

49. Bañares, n. 25, vol. 2, p. 78.

50. Más datos en Bertomeu Sánchez, José Ramón. La censura gubernativa de libros científicos durante el reinado de José I en España (1808-1813). Hispania. 1994; 54 (188): 917-954, de donde proceden las citas en p. 933. 
se volvía a insistir en que la mayor parte de los médicos y farmacéuticos continuaban empleando la nomenclatura antigua:

«Aunque la nueva nomenclatura química es por todos respectos preferible á la antigua, (...) se observa que á pesar de estas ventajas no se ha extendido aun ni hecho tan general como convenia el uso de la nueva nomenclatura. Nótase esto particularmente en los profesores de medicina y cirugía, de los quales rarísimo es entre nosotros el que dexa de seguir en sus recetas el uso de la nomenclatura antigua, y lo mismo los boticarios» ${ }^{51}$.

La situación no era exclusiva de España. En Francia, y probablemente en muchos otros países, los farmacéuticos continuaron empleando su propia nomenclatura durante mucho tiempo, aunque, gracias a la introducción de cursos obligatorios de química en su formación, cada vez fue mayor el conocimiento de los nuevos términos. El mayor peso de la química en farmacia estuvo relacionado con los cambios en la profesión y la aparición de las nuevas escuelas de farmacia, que reemplazaron parcialmente el antiguo sistema gremial de formación, lo que también estuvo asociado con la polémica. De hecho, los dos principales defensores de la nueva nomenclatura química, Gutiérrez Bueno y Carbonell i Bravo, eran también partidarios de los cambios en la forma de enseñanza y control de la profesión farmacéutica ${ }^{52}$.

En 1816, como ya hemos señalado, el farmacéutico Caventou escribió una obra destinada a explicar la nueva nomenclatura química, que fue rápidamente traducida al castellano por Higinio A. Lorente. Caventou señalaba que su obra estaba dirigida tanto a los nuevos estudiantes de química como a las personas que «estudiaron la antigua, y no han podido continuar después» y señalaba como ejemplo el caso de los maestros farmacéuticos:

«Un profesor de farmacia que tendrá la mejor instrucció en ella, no podrá tal vez decir á su mancebo, como llamamos nosotros ahora al emético, porque ignorará lo que se ha adelantado en el conocimiento de los cuerpos

51. Gaceta de Madrid. 8 de abril de 1812, p. 400.

52. Dere, Anne Claire. La réception de la nomenclature reformée par le corps médical français. In: Bensaude-Vincent; Abbri, n. 2, p. 207-225. Simon, Jonathan. Chemistry, Pharmacy and revolution in France, 1777-1809. Aldershot and Burlington: Ashgate; 2005, p. 189. Sobre la situación en España, veáse Bertomeu Sánchez, José Ramón; García Belmar, Antonio. Pedro Gutiérrez Bueno y las relaciones entre la química y la farmacia durante el último tercio del siglo XVIII. Hispania. 2001; 56 (2): 539-562. 
que examina la química, y las alteraciones que necesariamente han debido hacerse en el lenguage» 53 .

El caso del «emético» que menciona Caventou es suficientemente significativo. Se trataba de una sustancia con un amplio uso en farmacia que era nombrada habitualmente por sus propiedades médicas («emético»). El nombre químico que proponía Caventou («deuto-tartrato de potasio y de protóxido de antimonio») presentaba similares problemas a la expresión «sulfato ácido de alúmina y potasa» sugerida por Garriga y San Cristóbal para el «alumbre» en su obra de «química aplicada a las artes» ${ }^{54}$. Eran nombres demasiados largos y poco significativos a efectos prácticos, tanto para artesanos como para boticarios. Resulta comprensible, por lo tanto, que expresiones como «emético» o «tártaro emético» hayan sobrevivido al paso del tiempo hasta la actualidad.

Otra dificultad existente para la difusión de los nuevos términos era, paradójicamente, el criterio fundamental de la nueva nomenclatura: la composición química. Un primer problema era la posible confusión que podía provocar la nueva nomenclatura entre dos sustancias con propiedades terapéuticas muy diferentes pero composición semejante, tal y como ocurría, por ejemplo, con los nombres modernos de los cloruros del mercurio. En este caso, las nuevas voces se diferenciaban por prefijos o sufijos, lo que no ocurría en la nomenclatura antigua, en la que se designaban con nombres muy diferentes («sublimado corrosivo» y «calomelanos»), basada en propiedades médicas y físicas. Manuel Jiménez se refería en su terminología farmacéutica a las peligrosas equivocaciones que podían producir esta situación, dado que médicos y cirujanos podían poner «en sus recetas» nombres que «algunas veces equivalen científicamente a sustancias muy diferentes de las que era su intencion prescribir» ${ }^{55}$. Otro problema adicional era que la composición química de los productos farmacéuticos no siempre era definida ni conocida con precisión. Esta situación se daba particularmente en el caso de las sustancias de origen vegetal y animal pero también existen ejemplos de productos inorgánicos. En realidad, el cambio en el modo de

\footnotetext{
53. Caventou, n. 33, p. vi.

54. San Christóbal; Garriga y Buach, n. 45, vol. 1, p. 152.

55. Jiménez, n. 35, p. xv. Jiménez también indicaba que su obra debía ser útil a «los profesores de Farmacia (...) para que los practicantes puedan salir de las dudas que ofrezcan las recetas, y no equivoquen los nombres que a veces son muy semejantes en la apariencia aunque diversísimos en su composicion».
} 
nombrar suponía, en muchos casos, una modificación en la naturaleza ontológica de los materiales designados. Un ejemplo es el término «azafrán de marte», un producto de amplio uso en farmacia y que se presentaba en diversas variedades. Como muchos otros nombres antiguos, estaba basado en la similitud con el color (azafrán) y las relaciones alquímicas del hierro (marte). Su traducción a la moderna nomenclatura química no era sencilla porque su procedimiento de fabricación daba como resultado lo que actualmente consideraríamos una mezcla de óxidos y carbonatos de hierro. Existían diversas variedades cuya composición dependía de cuestiones como el método de preparación o de conservación y no todos los autores estaban de acuerdo respecto a la composición química de este producto farmacéutico ${ }^{56}$. En otras palabras, los términos farmacéuticos tradicionales, por lo tanto, no designaban solamente sustancias químicamente puras sino también mezclas obtenidas por procedimientos de preparación que, en ocasiones, podían tener carácter local.

Como hemos señalado anteriormente, otro problema de los nombres basados en la composición es que estaban sometidos a constantes revisiones, a medida que el avance de la química permitía conocer nuevas sustancias. Por ejemplo, si se descubría un nuevo óxido, debían reescribirse los nombres de los óxidos «proto-», «deuto-», etc. Si se reconsideraba el carácter elemental o compuesto de una sustancia, su nombre debía adaptarse para seguir los criterios generales de la nomenclatura. Esta alta variabilidad no se daba en nombres antiguos, basados en propiedades visibles como el color o en criterios más o menos convencionales, sin referencia explícita a ninguna propiedad, tales como epónimos o topónimos. Sobre esta cuestión se produjo una discusión con la llegada de los nuevos nombres para los derivados del ácido muriático, como consecuencia de la aceptación del carácter elemental del cloro, un tema polémico durante algunos años ${ }^{57}$. Gregorio Bañares, siguiendo un punto de vista semejante al de algunos autores británicos, señaló los riesgos de conectar la terminología con hipótesis no plenamente confirmadas, lo que podía dar lugar a frecuentes

56. Veáse por ejemplo, Soubeiran, Eugène. Nuevo tratado de farmacia teórico y práctico. Madrid: Boix; 1845-1846, vol. 4, p. 43. El asunto de la composición del azafrán de marte fue debatido en los años iniciales del siglo XIX. Lecciones elementales, n. 27, vol. 3, p. 109.

57. Chang, Hasok; Jacson, Catherine. An element of controversy: The life of chlorine in science, medicine, technology and war. London: BSHS; 2007, p. 407. 
modificaciones y a la multiplicación de los términos ${ }^{58}$. Bañares dudaba del carácter elemental del cloro («atendiendo a las propiedades maravillosas que se le atribuyen») y se preguntaba sobre las consecuencias de fundamentar la terminología en una propiedad que podía ser objeto de revisión con nuevas investigaciones:

«el cloro, repito, es considerado en el día como un cuerpo simple; pero esta simplicidad es inconcebible atendiendo á las propiedades maravillosas que se le atribuyen, y á que esta asercion hipotética y gratuita carece de demostraciones y hechos positivos que la apoyen [...] Ahora bien ¿será suficiente una hipótesis que no se funda en hechos demostrables, y que no destruye ninguna de las teorías antiguas del gas muriático oxigenado, considerado como tal; bastará, digo, para echar por tierra una nomenclatura la mas fundada y admitida generalmente hasta ahora? Nada menos que eso. Por el contrario, la considero muy perjudicial para su trascendencia; porque á imitacion suya se formarán todos los años bajo nuevas hipótesis nuevas teorías, y por consiguiente nuevos nombres, y el estudio de esta importante ciencia se reducirá principalemnte á tener en la memoria una multitud de voces abstracta» ${ }^{59}$.

A pesar de esta crítica general a los principios de la nueva nomenclatura, los farmacéuticos como Gregorio Bañares aceptaron los nuevos términos, en ocasiones, realizando sugerencias como las que hemos visto anteriormente y, por lo general, manteniendo las voces antiguas junto con las nuevas expresiones. La situación se mantuvo durante el siglo XIX ${ }^{60}$. En 1875, el farmacéutico y prolífico autor y traductor de libros de química, Rafael Sáez Palacios todavía mencionaba las resistencias a la nueva nomenclatura y señalaba las razones que las habían provocado. Por un lado, la existencia de sustancias con propiedades terapéuticas muy diferentes (cloruros del mercurio) cuyo nombre era muy semejante en la nueva nomenclatura, lo que podía dar lugar a confusiones funestas, que no se daban con los nombres

58. Sobre esta cuestión, veáse. Golinski, Jan. Science as public culture: Chemistry and Enlightenment in Britain, 1760-1820. Cambridge: University Press; 1992, p. 148-151.

59. Bañares, n. 34, p. 17-18.

60. Véase, por ejemplo, el popular manual de Ramón Capdevila, Elementos de terapéutica y materia médica. $6^{\text {a }}$ ed. Madrid: Fuentenebro; 1843. Aunque se ofrecen siempre los nombres modernos en los apartados correspondientes, muchas expresiones antiguas son mayoritariamente utilizadas en las explicaciones de los diversos productos y operaciones, por ejemplo, «aceite de vitriolo» (p. 31), «vitriolo azul» (p. 207), «albayalade» (p. 207-208), «sal de la higuera» (p. 419), lo que indica su amplio uso en esos años. 
antiguos. Estas confusiones, pensaba Sáez Palacios, se daban más en el caso de los médicos que debían recetar, porque apenas tenían conocimientos de química. En otras palabras, la nueva nomenclatura química exigía muchos más conocimientos de esta ciencia que los nombres tradicionales y, por lo tanto, como hemos visto, su difusión estuvo asociada al nuevo modelo de enseñanza que se consolidó en las facultades de farmacia del siglo XIX. Finalmente, Sáez Palacios también señalaba las frecuentes modificaciones de la nomenclatura química moderna, debido a las causas que hemos revisado en los apartados anteriores ${ }^{61}$.

\section{Conclusiones}

La discusión anterior nos ha permitido recuperar aspectos de la introducción de la nueva terminología que fueron muy relevantes para los autores de manuales de química pero apenas tomados en cuenta hasta la fecha por los historiadores. En primer lugar, hemos visto la existencia de fuertes controversias en torno al modo en el que se debían adaptar las nuevas expresiones al castellano. Algunos autores abogaron por mantener los términos con escasas modificaciones, con el objetivo de mantener un lenguaje científico lo más universal posible. Otros, por el contrario, destacaron la necesidad de adaptar las nuevas expresiones a la naturaleza de la lengua de acogida, aunque el resultado fuera muy diferente al original francés. Entre estas dos posturas extremas, existieron toda una serie de situaciones particulares que estuvieron moldeadas por los intereses del autor y de sus públicos destinatarios, así como por las características particulares de los términos, tal y como hemos visto en el caso de la expresión «azote», con los problemas de homonimia ya señalados. En otros casos, el importante peso de las traducciones francesas acabó imponiendo una forma («sulfuro») en detrimento de otra («sulfureto») que había sido inicialmente adoptada sin controversias por toda la comunidad. El resultado fue una gran variedad terminológica que quedó plasmada en los libros de texto con diversas formas para varios nombres de sustancias que alcanzaron una vida más o menos larga. Hubo expresiones que desaparecieron en los manuales de química

61. Saéz Palacios, Rafael. Tratado de química inorgánica teórico y práctico, aplicada a la medicina y especialmente a la farmacia. $2^{\text {a }}$ ed. Madrid: Carlos Bailly Baillière; 1875, p. 149-150. 
pero que perduraron en obras de farmacia o medicina. Esta diversidad terminológica estuvo acentuada por la constante renovación del vocabulario químico durante la primera mitad del siglo XIX, de modo que fue necesario realizar modificaciones y ampliaciones de las reglas establecidas a finales del siglo anterior. Las modificaciones fueron acogidas, por lo general, con comentario críticos y, en algunas ocasiones, con propuestas originales, tal y como hemos visto en el caso de Gregorio Bañares y Antonio Chalanzón. En cualquier caso, la terminología sugerida por los autores franceses fue la predominante en los manuales hasta los años treinta y cuarenta del siglo XIX, cuando comenzó a introducirse la nueva nomenclatura sugerida por Jöns Jacob Berzelius.

Hemos comprobado que la llegada de la nueva terminología no supuso la desaparición inmediata de las expresiones antiguas. Éstas convivieron durante mucho tiempo con la nueva nomenclatura. Para conocer estas resistencias y permanencias resulta imprescindible profundizar en el conocimiento de la química del siglo XVIII en España para repensar las consecuencias de la denominada «revolución química» y ampliar el número de protagonistas para conocer con detalle los diversos puntos de vista a traves de las numerosas controversias que se produjeron. Hemos comprobado que el mantenimiento de expresiones antiguas estuvo justificado por las características de áreas como la farmacia y la medicina, donde las ventajas de los nuevos nombres basados en la composición química resultaban menos evidentes. La antigua nomenclatura estaba formada por términos de frecuente uso, más breves y menos susceptibles de prestarse a confusiones. Además, la persistencia de voces antiguas permitía evitar los problemas generados por los cambios constantes en la nueva terminología, provocados por el descubrimientos de nuevas sustancias o por nuevas investigaciones sobre su composición, tal y como recordaba Bañares en el caso del cloro.

Nuestro análisis también muestra el interés que tiene la terminología para repensar las relaciones entre centros y periferias científicas y para analizar los mecanismos de apropiación que suelen acompañar el movimiento de la ciencia. El proceso de aclimatación de las nuevas expresiones estuvo condicionado por aspectos como las tradiciones locales de enseñanza -en el caso de Joseph-Louis Proust y la pervivencia de sus expresiones para los óxidos- o por las comunidades profesionales a las que se dirigían las obras, tal y como se ha visto en el caso de los boticarios. En este sentido, la progresiva implantación de la nueva terminología estuvo íntimamente ligada con los cambios en la profesión farmacéutica durante el siglo XIX, el abandono 
del antiguo sistema gremial y la consolidación de un modelo de enseñanza en el que la química y la historia natural jugaban un papel destacado. No es casual que los defensores de la nueva terminología química fueran también firmes partidarios de este proceso de redefinición de la farmacia. También hemos constatado la existencia de resistencias y propuestas alternativas por parte de muchos boticarios que aprovecharon la publicación de manuales o, incluso, estudios sobre análisis de aguas para hacer públicos sus puntos de vista. Sus propuestas tuvieron un eco muy escaso, en parte por su limitada capacidad para difundirlas más allá de la comunidad a la que pertenecían. No obstante, sus reacciones son suficientemente informativas de la gran variedad de posturas y planteamientos con los que fue acogida la nueva nomenclatura, que incluyeron desde la aceptación crítica hasta la resistencia parcial o total, bien mediante la defensa de voces antiguas o a través de la propuesta de nombres alternativos. La riqueza de estos planteamientos no puede describirse con la dicotomía de supuestos partidarios y detractores de las nuevas ideas, una contraposición demasiado manida por los polemistas de la ciencia española y sus epígonos. Superada esta simplificación interesada, resulta posible ampliar el abanico de preguntas para transformar la historia de la terminología química en una vía para comprender mejor los procesos de circulación de la ciencia. 